\title{
Antibacterial Effect of Human Milk against Streptococcus pyogenes: an in vitro Study
}

\author{
Novi Vicahyani Utami, ${ }^{1}$ Nurul Nadiya Binti Abdul Razak, ${ }^{2}$ Dzulfikar D. L. Hakim ${ }^{3}$ \\ ${ }^{1}$ Department of Biomedical Sciences Faculty of Medicine Universitas Padjadjaran, Indonesia, \\ ${ }^{2}$ Faculty of Medicine Universitas Padjadjaran, Indonesia, ${ }^{2}$ Department of Child Health Faculty of \\ Medicine Universitas Padjadjaran/Dr. Hasan Sadikin General Hospital Bandung, Indonesia
}

\section{Abstract}

Background: Streptococcus pyogenes infection is one of the common upper respiratory infections among children. Human milk has antibacterial properties that may play a role against infections. The study was conducted to observe the antibacterial effect of human milk against Streptococcus pyogenes.

Methods: This was an in vitro experimental study conducted on October-November 2012 in Microbiology Laboratory, Faculty of Medicine, Universitas Padjadjaran, Bandung, using agar well diffusion method. Human milk was collected from eligible donors and tested at concentrations of $70 \%, 80 \%, 90 \%$, and $100 \%$ dilution with water. Inhibition zone formed surrounding the wells were measured after 24 hours of incubation.

Results: All samples incubated with human mils (in concentration of $70 \%, 80 \%, 90 \%$ and $100 \%$ ) inhibited the growth of Streptococcus pyogenes, with the inhibition zone mean diameters of 26.33, $28.00,28.00$ and $28.33 \mathrm{~mm}$, respectively, compared to the well containing sterile aquadest that did not show any zone of inhibition.

Conclusions: Human milk has an in vitro antibacterial effect against Streptococcus pyogenes. Giving human milk to babies is important for preventing infectious diseases.

Keywords: Antibacterial, human milk, in vitro, Streptococcus pyogenes

\section{Introduction}

Infectiousdiseaseisoneofthemajorglobalburden of diseases worldwide. ${ }^{1}$ Infectious diseases or commonly known as communicable diseases are caused by pathogenic microorganisms, such as bacteria, viruses, fungi or parasites. It is easily transmitted from one person to another, either by airborne, blood-borne, food-borne, sexual contact, skin contact and many others. ${ }^{2}$ Various transmission pathways could be the reasons why these diseases are common and cause high mortality and morbidity globally. The Global Burden of Disease 2004 Report has shown that infectious and parasitic diseases ranked second as the leading cause of death worldwide. Such diseases caused almost 16\% of all deaths in women and $17 \%$ in men. It is also reported that infections and parasitic diseases are the major causes of death in the low-income countries such as Africa and Southeast Asia with approximately $34 \%$ of total deaths. ${ }^{1}$

Among the infectious diseases, bacterial diseases are the major health problems for humans. Tuberculosis for example still ranks among the world's leading causes of death. ${ }^{3}$ Group A Streptococcus (GAS) disease is another bacterial disease that is reported as a global disease burden in the World Health Organization (WHO) report in 2004.; about 18.1 million people had suffered from a serious GAS disease at that time, and these diseases are responsible for over 500,000 deaths each year. ${ }^{4}$ Group A Streptococcus, also known as Streptococcus pyogenes is the common bacterial pathogen that is responsible for a broad spectrum of diseases that range from mild sore throat until life-threatening

Correspondence: Novi Vicahyani Utami, Department of Biomedical Sciences Faculty of Medicine Universitas Padjadjaran, Jalan Raya Bandung Sumedang Km. 21 Jatinangor, Sumedang Indonesia , E-mail: novi.farmakologi@gmail.com 
illnesses like necrotizing fasciitis. ${ }^{5}$ This highly transmissible bacterium also a common cause of upper respiratory and skin infections. A study conducted in Chennai ${ }^{6}$ has shown that the prevalence of Group A streptococcus was high $(36 \%)$ compared to other groups of streptococcus among the slum children.

Many clinicians have to confront this GAS infection every day, as this is not only an acute illness for a single individual patient but also as a public health issue related to the highest potential to spread. Vaccines to combat these Streptococcus pyogenes also are not yet available. Penicillin has been used for many years to treat this infection, however, many bacteria are now resistant to penicillin. ${ }^{7}$

Human milk has known worldwide for its beneficial values towards infants. It is a very good source of nutrients, and it has immunological properties. A study in the United State $^{8}$ has reported that exclusively breastfed babies have a decrease in respiratory tract infections. This might be contributed to the anti-infective properties in the human milk, which cannot be found on other foodstuffs; such as lactoferrin, secretory IgA, $\alpha$-amylase, lysozymes, defensins, cathelicidins and many others that have antimicrobial activity. ${ }^{9-11}$ Therefore, human milk could be used as an alternative to combat infection.

Many studies confirm that human milk has antimicrobial activity against pathogens, among others in children with diarrhea. ${ }^{12}$ This study aimed to explore the antimicrobial activity of human milk against Streptococcus pyogenes.

\section{Methods}

This research was an experimental study, conducted in October-November 2012 in the Microbiology Laboratory, Faculty of Medicine, Universitas Padjadjaran, Bandung, Indonesia.

Human milk samples were collected from donors that fulfilled the inclusion criteria after having written informed consent. The inclusion criteria of the donors were healthy nursing mothers beyond four weeks postpartum, delivered to healthy full-term infants, and with no intake of medications within one week before collection day. Human milk samples were collected manually into a sterile human milk storage bags and stored in the refrigerator at $4^{\circ} \mathrm{C}$ until used the next day. For this research, human milk was used as undiluted (100\%) and at 70,80 and $90 \%$ dilutions.

Streptococcus pyogenes were obtained from Microbiology Laboratory, Faculty of Medicine, Universitas Padjadjaran. The bacteria were harvested from a confluent streaked petri dish and suspended in sterile $\mathrm{NaCl} 0.9 \%$ to match the 0.5 McFarland standards. The bacteria suspension was then mixed with MuellerHinton broth and 5\% sheep blood to make inoculated Mueller-Hinton blood agar plate.

The antibacterial effect of human milk against Streptococcus pyogenes was tested using the agar well diffusion method. Human milk samples were diluted with sterile aquadest at concentrations of $70 \%, 80 \%, 90 \%$, and $100 \%$. The inoculated Mueller-Hinton blood agar was prepared by making wells on it using a sterile well borer. One hundred and
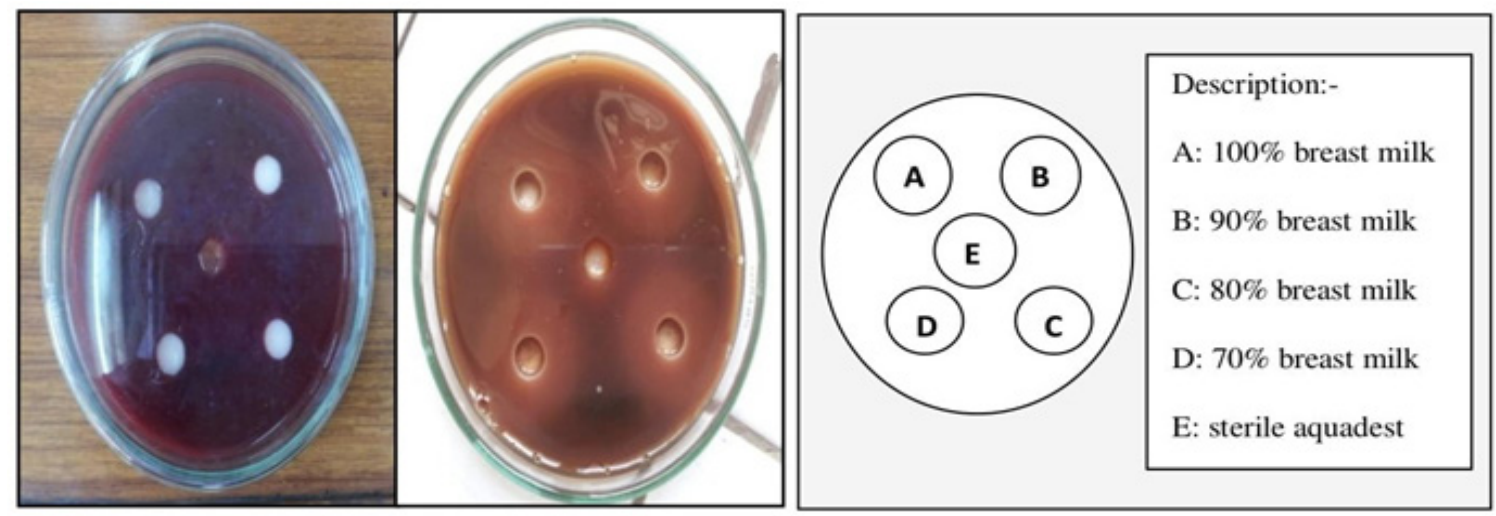

Figure Zone of Inhibition of Human Milk against Streptococcus pyogenes. 
Table Diameter of Zone of Inhibition of Human Milk against Streptococcus pyogenes

\begin{tabular}{lccccc}
\hline \multirow{3}{*}{ No. of replication } & \multicolumn{5}{c}{ Concentrations } \\
\cline { 2 - 6 } & $\mathbf{1 0 0 \%}$ & $\mathbf{9 0 \%}$ & $\mathbf{8 0 \%}$ & $\mathbf{7 0 \%}$ & Control \\
\cline { 2 - 6 } & 28.00 & 28.00 & 28.00 & 26.00 & 0.00 \\
2 & 28.00 & 28.00 & 28.00 & 27.00 & 0.00 \\
3 & 29.00 & 28.00 & 28.00 & 26.00 & 0.00 \\
$\bar{x}$ & 28.33 & 28.00 & 28.00 & 26.33 & 0.00 \\
\hline
\end{tabular}

sixty microliters of different concentrations of human milk and sterile aquadest were dispensed into the wells made and incubated at $37^{\circ} \mathrm{C}$ for 24 hours. The diameter of the inhibition zones formed surrounding the wells were observed and measured in millimeter $(\mathrm{mm})$. The inhibition zones were compared with the inhibition zone of the well containing sterile aquadest that was served as a negative control. The antibacterial test was performed in triplicates.

\section{Results}

The inhibition zone was formed surrounding the well that dispensed with human milk samples. The well that contained sterile aquadest did not show any zone of inhibition, as shown in Figure.

After three times replication of the antibacterial test, the human milk with a dilution of $70 \%, 80 \%, 90 \%$, and $100 \%$ showed zones of inhibition; whereas the sterile aquadest as a negative control showed no zone of inhibition. The diameters of the inhibition zones were presented in Table.

The mean diameter of the zone of inhibition formed was slightly different between each dilution used, however, no statistical difference among those dilutions. The $100 \%$ of human milk has shown the best antibacterial effect against Streptococcus pyogenes of all the concentrations tested with the highest mean diameter of $28.33 \mathrm{~mm}$.

\section{Discussion}

The result of this research has proved that human milk can inhibit the growth of the Streptococcus pyogenes in vitro. There are many components in human milk that may be responsible for this antibacterial effect. Protection from infection by human milk is provided by two mechanisms. First, the production of non-specific protective factors that is independent of exposure to infectious or other foreign agents like lactoferrin, lysozyme, gluco-conjugates, and oligosaccharides. Second, the antigen-dependent mechanism which leads to the production of secretory IgA antibodies that protect against enteric and respiratory pathogens. ${ }^{9}$

The secretory IgA is believed to be the most influencing factor in human milk which has the antibacterial effect against Streptococcus pyogenes. Secretory IgA is the main immunoglobulin present in human milk. It can attach to the mucosal epithelium of the gut and some parts of the respiratory tract, and it prevents the attachment and invasion of specific infectious agents. ${ }^{10}$ The secretory IgA itself has been proven to have an in vitro antibacterial effect against Streptococcus pyogenes. ${ }^{11}$

The non-specific protective factors may also contribute to the antibacterial effect of human milk. Lactoferrin, a protein in human milk can demonstrate a bacteriostatic effect. Milk lysozyme which can cleave peptidoglycans of the bacterial cell walls has been known to have a higher concentration in human milk. Other factors like oligosaccharides and glycoconjugates have been also known to inhibit the adherence and proliferation of bacteria on the epithelial surfaces. ${ }^{10}$ All of the non-specific factors have shown in vitro activity against common pathogens like Staphylococcus aureus, Escherichia coli, Vibrio cholera and Haemophilus influenzae. ${ }^{11}$

Aprevious studyusingprobiotic of the human milk has shown that there is an antibacterial activity against common pediatric communityacquired respiratory pathogens. The probiotic of the human milk can inhibit the growth of Streptococcus pneumonia, Haemophilus influenzae and Staphylococcus aureus tested in this study, ${ }^{13}$ thus, confirming that the human 
milk can prevent the growth of bacteria which causes upper respiratory infections, including the Streptococcus pyogenes.

There is another study reporting the exclusively breastfed infants who have less hospitalization caused by respiratory tract illness compared to non-breastfed infants. ${ }^{14}$ The study has portrayed the best of the protective role of human milk, also supported the proposed mechanisms of anti-infective components of the human milk in protecting against pathogens.

The result of this research further has proved the protective role of human milk against common respiratory pathogens. The antibacterial effect of the human milk against Streptococcus pyogenes is beneficial as some studies have shown that Streptococcus pyogenes develop a resistance to some antibiotics. New alternative treatment for Streptococcus pyogenes infections is needed especially in children. Furthermore, human milk convenient to be used as human milk is a less cost, safe and natural source of treatment options for the community. This study has limitations not to compare the antibacterial effect against Streptococcus pyogenes between human milk and formula milk. Further study may be of great interest to explore the antibacterial effect of the human milk above the formula milk.

In conclusion, human milk has an in vitro antibacterial effect against Streptococcus pyogenes. It is thus important to give human milk to the infant for preventing infectious diseases.

\section{References}

1. World Health Organization. The global burden of disease: 2004 update. Geneva: WHO Press; 2008.

2. Lucile Packard Children's Hospital Standford. Maternal and fetal infections overview. [Online Web Page] 2010 [Cited 2012 April 26]; Available from: https:// www.stanfordchildrens.org/en/topic/ default?id=maternal-and-fetal-infectionsoverview-90-P09523\&sid=.

3. World Health Organization. World health statistics 2011. Geneva: WHO Press; 2011.

4. World Health Organization. The current evidence for the burden of group A Streptococcal diseases. Geneva: WHO Press; 2005.

5. MedlinePlus. Bacterial infections. [Online Web Page] 2019 [Cited 2019 October 13]; Available at: https://medlineplus.gov/ bacterialinfections.html.

6. Kalpana S, Sundar JS, Parameshwari S, Kuganantham P, Selvam JM, Valarmathi MS, et al. Isolation and identification of group A Streptococcal infection among slum children in the age group of 5-15 years in Chennai-one year prospective study. IOSRJPBS. 2012;2(1):27-30.

7. Pechere JC, Kaplan EL, editors. Streptococcal pharyngitis: optimal management. Basel, Switzerland: Karger Medical and Scientific Publishers; 2004. p. 16-32.

8. Chantry CJ, Howard CR, Auinger P. Full breastfeeding duration and associated decrease in respiratory tract infection in US children. Pediatrics. 2006;117(2):42532.

9. Goldman AS. The immune system in human milk and the developing infant. Breastfeed Med. 2007;2(4):195-204.

10. Lonnerdal B. Nutritional and physiologic significance of human milk proteins. Am J Clin Nutr. 2003;77(6):1537S-43S.

11. Aldy OS, Lubis BM, Sianturi P, Azlin E, Tjipta GD. Dampak proteksi air susu ibu terhadap infeksi. Sari Pediatri. 2009;11(3):167-73.

12. Chan GM, Lee ML, Rechtman DJ. Effects of a human milk-derived human milk fortifier on the antibacterial actions of human milk. Breastfeed Med. 2007;2(4):205-8.

13. Cid SRC, Cruz MC, Faustino V, Tuazon AO. In vitro study on the antimicrobial activity of probiotic milk against common pediatric community acquired respiratory pathogens. PIDSP Journal. 2005;9(2):259.

14. Bachrach VRG, Schwarz E, Bachrach LR. Breastfeeding and the risk of hospitalization for respiratory disease in infancy: a meta-analysis. Arch Pediatr Adolesc Med. 2003;157(3):237-43. 